$\Rightarrow$ BASIC RESEARCH

\title{
Inhibition of Hippo pathway signalling reverses systolic heart failure
}

Given the poor regenerative capacity of the adult heart, patients who survive the acute phase of myocardial infarction often develop heart failure. During heart failure, compensatory pathological remodelling leads to further cardiomyocyte fibrosis and death. Maladaptive activation of the

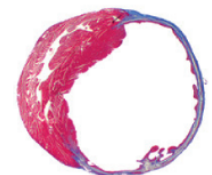

Control heart

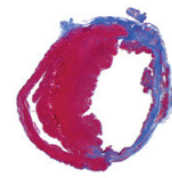

SalvCKO heart

Reprinted from Leach, J. P. et al. Hippo pathway deficiency reverses systolic heart failure after infarction. Nature http://dx.doi.org/10.1038/ nature24045 (2017) @ Macmillan Publishers Limited.

Hippo pathway, an evolutionarily conserved regulator of organ growth, occurs during both ischaemia and reperfusion injury in mice, and in heart failure in humans. In a new study published in Nature, Leach et al. report that deletion of the Hippo pathway component Salvador (Salv) in a mouse model of post-infarction heart failure resulted in cardiomyocyte renewal and reversal of the heart failure phenotype.

Leach and colleagues generated mice with conditional knockout of Salv (SalvCKO), with ischaemic heart failure after myocardial infarction. Mice aged 8-10 weeks underwent either a sham or a myocardial infarction procedure. Compared with the sham mice, those mice that underwent left anterior descending coronary artery ligation showed features of impaired systolic function on echocardiography, including reduced ejection fraction, reduced fractional shortening, and increased systolic left ventricular chamber diameter and volume. Other features of heart failure in these mice included build-up of pulmonary fluids, increased serum levels of B-type natriuretic peptide, and elevated body weight. Salv was deleted in cardiomyocytes 3 weeks after the procedure.

Compared with control hearts, SalvCKO hearts at 9 weeks after myocardial infarction had a higher number of cardiomyocytes in the left ventricle $\left(1 \times 10^{5} \pm 8 \times 10^{4}\right.$ versus $\left.6 \times 10^{5} \pm 2 \times 10^{5}\right)$ and less fibrosis (percentage area fibrosis: $56 \pm 16 \%$ versus $36 \pm 15 \%$ ). Furthermore, at 6 weeks after myocardial infarction, SalvCKO hearts had a threefold increase in border zone capillary density, as well as increased expression of vasculogenesis genes that encode angiopoietins, fibroblasts growth factors, and vascular endothelial growth factors.

Cardiomyocyte-enriched translating ribosomal affinity purification (TRAP) RNA sequencing of SalvCKO and control hearts after myocardial infarction revealed 365 upregulated genes in the SalvCKO hearts, including cell cycle genes and genes involved in heart contraction, heart growth, and cellular response to stress. A total of 261 genes were downregulated in the SalvCKO cardiomyocytes compared with control cardiomyocytes, which included those involved in protein translation and metabolism.

"Hippo pathway inhibition ... results in an effective stress response in ischaemic heart failure with organ failure reversal," the investigators conclude. "Since Hippo signalling is upregulated in human ischaemic heart failure, Hippo pathway inhibition may have a therapeutic benefit for patients with ischaemic heart failure."

Junichi Sadoshima (Rutgers New Jersey Medical School, USA), who was not involved in the study, welcomes these findings, but cautions that because Hippo pathway inactivation might promote tumorigenesis in other organs, "interventions to modulate the Hippo pathway in a cardiomyocyte-specific manner need to be explored".

Karina Huynh

ORIGINAL ARTICLE Leach, J. P. et al. Hippo pathway deficiency reverses systolic heart failure after infarction Nature http://dx.doi.org/10.1038/nature24045 (2017) 